\title{
Transferability of Dipole Moment Derivatives: A General Method for the Calculation of Absolute Rotational Contributions
}

\author{
W. M. A. Smit*, J. H. G. Bode, and A. J. van Straten $\dagger$ \\ Analytical Chemistry Laboratory, University of Utrecht, \\ Croesestraat 77A, 3522 AD Utrecht, The Netherlands
}

\begin{abstract}
A general method for the calculation of absolute rotational corrections for the dipole moment derivatives is given based on the definition of pure geometrical distortions. The method is generally valid and allows the rotational contributions to be calculated for all modes of any molecule. The relation between absolute and relative corrections is discussed and the method is demonstrated by calculating the rotational corrections for a few asymmetrical molecules.
\end{abstract}

\section{INTRODUCTION}

The infrared intensities of corresponding modes in different molecules are not directly comparable. This is because, in general, the conditions which restrain the principal-axis system from rotation during vibration work out different for corresponding modes in different molecules. These conditions are usually called the Eckart conditions. In order to obtain comparable intensities the dipole moment derivative values should be split up in two parts, one corresponding to a pure geometrical distortion, the other to the compensating rotation that has to be added to the geometrical distortion in order to satisfy the Eckart conditions. As already shown by Polo $(l)$ in 1956 a pure geometrical internal coordinate distortion can be chosen in an infinite number of ways. However, in the previous paper (2) we have shown that absolute rotational contributions can be calculated for small symmetrical molecules since the pure geometrical distortions can be uniquely defined for all modes of these molecules.

In the present paper the general relationships between pure geometrical reference coordinates and the rotational contributions involved in the dipole moment derivatives will be discussed. To demonstrate the method the rotational corrections for a number of asymmetrical molecules will be given.

\section{THEORETICAL CONSIDERATIONS}

The definition of the pure geometrical internal coordinates $(l)$ implies

$$
\mathbf{B} \mathbf{A}_{\mathrm{g}}=\mathbf{E} .
$$

* Author to whom correspondence should be addressed.

$\dagger$ Present address: Koninklijke/Shell Laboratorium (Shell Research B.V.), Badhuisweg 3, Amsterdam, The Netherlands. 
Making use of the condition (3)

one easily arrives at

$$
\mathbf{A B}+\boldsymbol{\alpha} \boldsymbol{\beta}=\mathbf{E}_{3 N, 3 N},
$$

$$
A=A_{g}-\alpha \beta A_{g},
$$

which is equivalent to Eq. (II, 8) of Polo's paper (I). Equation (3) corresponds closely to the relation between the A matrices belonging to different members of the same isotopic family as given by Crawford (4),

$$
\mathbf{A}_{1}=\mathbf{A}_{2}-\alpha_{1} \boldsymbol{\beta}_{1} \mathbf{A}_{2},
$$

where the subscripts refer to the different members of the isotopic series. However, the $\mathbf{A}_{\mathrm{s}}$ matrix cannot be considered as the $\mathbf{A}$ matrix belonging to a hypothetical member of the series, since $\mathbf{A}_{\mathrm{g}}$ corresponds to pure geometrical coordinates which are independent of the atomic masses. The one $\mathbf{A}_{\mathrm{g}}$ matrix applies to all members of the isotopic series if the same Cartesian axis system is used for all molecules.

As pointed out in our previous paper (2), Eq. (3) leads to the following expressions for the absolute rotational contributions in terms of internal coordinates:

$$
\begin{aligned}
\mathbf{P}_{\mathrm{R}} & =\mathbf{P}_{\mathrm{Rg}}+\mathbf{V}, \\
\mathbf{V} & =-\mathbf{P}_{\rho} \boldsymbol{\beta} \mathbf{A}_{\mathrm{Rg}} .
\end{aligned}
$$

The polar tensor $\mathbf{P}_{\mathrm{R}}$ contains the observed dipole moment derivatives, the $\mathbf{P}_{\mathrm{Rg}}$ tensor contains the dipole moment derivatives in terms of pure geometrical internal reference coordinates, while the rotational contributions are collected in the polar tensor $\mathbf{V}$.

For a given molecule, $\mathbf{P}_{\rho}$ is completely determined by the magnitude of the permanent dipole moment and its position in the principal-axis system, while the $\boldsymbol{\beta}$ matrix elements are given by the masses and geometrical parameters of the molecule (3). The elements of $\mathbf{A}_{\mathrm{Rg}}$ are the components of the pure geometrical Cartesian displacement vectors as defined by Polo $(1,2)$. In principle, the $\mathbf{A}_{\mathrm{Rg}}$ elements contain some arbitrariness since the pure geometrical displacement vectors (the $\rho$-vectors) can be chosen in an infinite number of ways. It has therefore to be investigated whether or not the elements of the $\boldsymbol{\beta} \mathbf{A}_{\mathrm{Rg}}$ product matrix which represent the amounts of rotation and translation set up in the molecule by the pure geometrical distortions depend on the actually chosen $\rho$-vectors. In the following this will be done for stretching and bending modes.

\section{Stretching Modes}

Let us consider the $\mathrm{OH}$ stretching mode of the bent triatomic molecule HOF. The Cartesian axes, bond unit vectors, and geometrical $\rho$-vectors are indicated in Fig. 1. The internal coordinates are:

$$
\begin{array}{ll}
R_{1}=\Delta r_{31}, & \text { the OH stretching mode, } \\
R_{2}=\Delta r_{32}, & \text { the OF stretching mode, } \\
R_{3}=\Delta \phi, & \text { the bending mode. }
\end{array}
$$


The Polo $\rho$-vectors for the $\mathrm{OH}$ stretching mode are

$$
\begin{array}{ll}
R_{1}: & \boldsymbol{\rho}_{11}=k_{11} \mathbf{e}_{31}, \\
& \boldsymbol{\rho}_{12}=\boldsymbol{\rho}_{13}=-k_{13} \mathbf{e}_{31},
\end{array}
$$

where the constants $k$ have to satisfy the relation

but are otherwise arbitrary.

$$
k_{11}+k_{13}=1
$$

The components of the $\rho$-vectors for $R_{1}$ can be derived to be

$$
\begin{aligned}
& \boldsymbol{\rho}_{11}=\left(-k_{11} \sin \alpha, 0,-k_{11} \cos \alpha\right), \\
& \boldsymbol{\rho}_{12}=\left(k_{13} \sin \alpha, 0, k_{13} \cos \alpha\right), \\
& \boldsymbol{\rho}_{13}=\left(k_{13} \sin \alpha, 0, k_{13} \cos \alpha\right),
\end{aligned}
$$

where $\alpha$ is the angle between $r_{31}$ and the $z$-axis. From the general form of the $\boldsymbol{\beta}$ matrix elements (3) and the above $\boldsymbol{\rho}$-vector components the element of the $\boldsymbol{\beta} \mathbf{A}_{\mathrm{Rg}}$ matrix describing the rotation around the principal $y$-axis caused by the pure geometrical $\mathrm{OH}$ stretching mode can be written as

$$
\begin{aligned}
\left(\beta A_{\mathrm{Rg}}\right)_{y 1} & =I_{y}^{-1} k_{11} m_{1}\left(x_{01} \cos \alpha-z_{01} \sin \alpha\right) \\
& -I_{y}^{-1} k_{13} m_{2}\left(x_{02} \cos \alpha-z_{02} \sin \alpha\right)-I_{y}^{-1} k_{13} m_{3}\left(x_{03} \cos \alpha-z_{03} \sin \alpha\right),
\end{aligned}
$$

where $x_{0 i}, y_{0 i}$, and $z_{0 i}$ are the components of $r_{0 i}$, the position vector of atom $i$ in the principal-axis system. Using Eq. (8) leads to

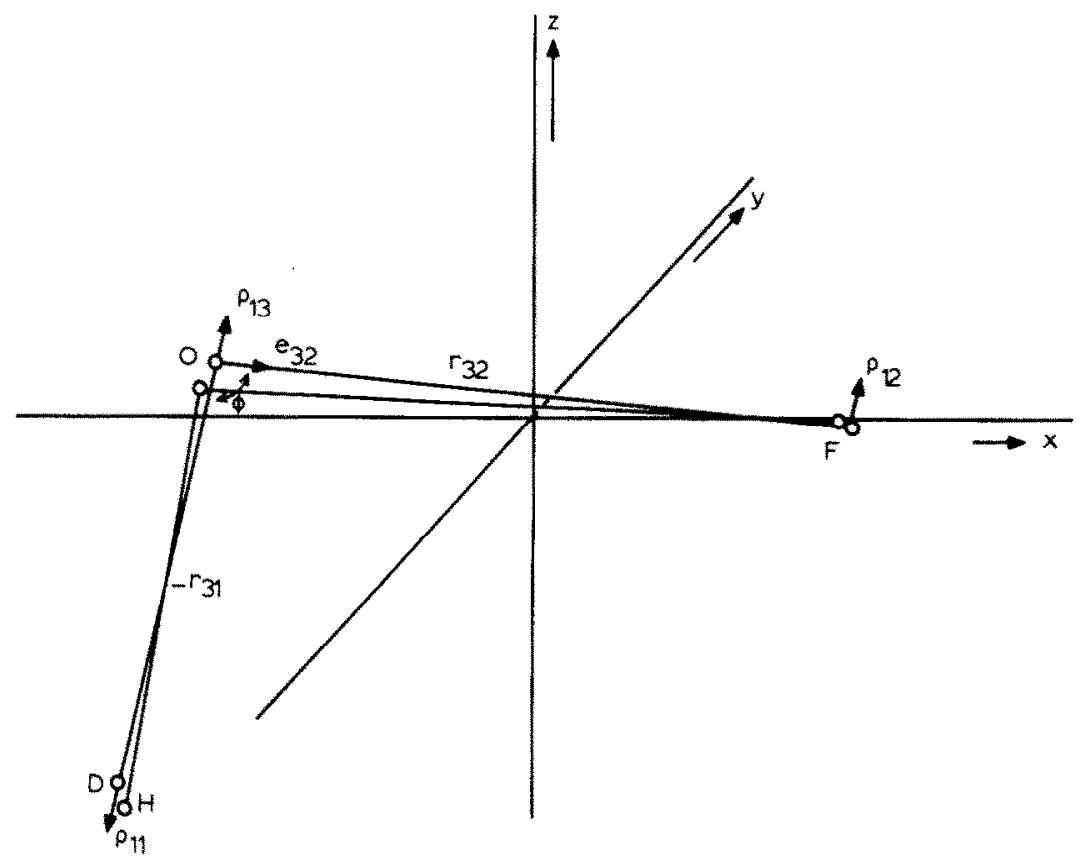

Fig. 1. Principal axes, internal coordinates, and bond unit vectors for HOF and DOF. 


$$
\begin{aligned}
\left(\beta A_{\mathrm{Rg}}\right)_{y 1}=I_{y}^{-1} m_{1}\left(x_{01} \cos \alpha-\right. & \left.z_{01} \sin \alpha\right) \\
& -I_{y}^{-1} k_{13}\left[\left(\sum_{i} m_{i} x_{0 i}\right) \cos \alpha-\left(\sum_{i} m_{i} z_{0 i}\right) \sin \alpha\right] .
\end{aligned}
$$

Since in the principal-axis system $\sum_{i} m_{i} x_{0 i}=\sum_{i} m_{i} z_{0 i}=0$, Eq. (11) appears to be independent of $k_{13}$. This leads to the conclusion that the rotational correction for the $R_{1}$ mode is independent of the arbitrariness in the pure geometrical displacements. It is easily seen that this is a general result for stretching modes, clearly showing the fundamental character of the rotational contribution involved in the dipole moment derivatives with respect to stretching coordinates.

\section{Bending Modes}

The components of the $\rho$-vectors for the pure geometrical bending mode $R_{3}$ of the HOF molecule are

$$
\begin{array}{ll}
R_{3}: & \rho_{31}=\left(-k_{31} r_{31} \cos \alpha, 0, k_{31} r_{31} \sin \alpha\right), \\
& \boldsymbol{\rho}_{32}=\left(k_{32} r_{32} \cos \beta, 0, k_{32} r_{32} \sin \beta\right), \\
\boldsymbol{\rho}_{33}=(0,0,0) .
\end{array}
$$

The rotation set up in the molecule by these displacements is given by

$$
\left(\beta A_{\mathrm{Rg}}\right)_{y 3}=-I_{y}^{-1} m_{1} r_{31}\left(x_{01} \sin \alpha+z_{01} \cos \alpha\right)-k_{32},
$$

where use has been made of the relations

$$
\begin{gathered}
r_{31} \sin \alpha=-x_{01}+x_{03}, \quad r_{31} \cos \alpha=-z_{01}+z_{03} ; \\
r_{32} \sin \beta=x_{02}-x_{03}, \quad r_{32} \cos \beta=-z_{02}+z_{03} .
\end{gathered}
$$

Obviously, Eq. (13) cannot be reduced to a form which is independent of $k_{32}$. This means that the rotational correction for $R_{3}$ depends on the $\rho$-vector displacements actually chosen, leading to the conclusion that no absolute rotational correction exists for the bending mode of this molecule. Although Eq. (13) is only valid for bent $X Y Z$ molecules, the resulting conclusion is generally valid.

A little reflection shows that the constants $k$ have a definite value, namely $k=1 / 2$, only in cases in which the bisectrix plane of the angle under consideration is a symmetry plane of the molecule. In such cases the corrections may be considered as absolute. In all other cases an arbitrary choice has to be made for the $k$ values in order to fix the amount of rotation set up in the molecule by the geometrical distortion. The resulting rotational corrections should then be considered as relative to the chosen geometrical reference modes. However, such corrections may better be denoted as semiabsolute in order to distinguish them from the relative corrections that are used to compare corresponding intensities within an isotopic series $(3-5)$. In view of the above remarks we propose to use $k=1 / 2$ throughout for all geometrical modes in any molecule. This leads to an easy, programmable, and consistent procedure for the calculation of rotational contributions that satisfies symmetry requirements if present. 


\section{Relative versus (semi-) Absolute Rotational Corrections}

As pointed out by Crawford (4) for the comparison of intensity data within an isotopic series, relative rotational corrections can be used. From Eq. (4) one easily obtains

$$
\mathbf{P}_{X} \mathbf{A}_{1}=\mathbf{P}_{X} \mathbf{A}_{2}-\mathbf{P}_{X} \boldsymbol{\alpha}_{1} \boldsymbol{\beta}_{1} \mathbf{A}_{2},
$$

where the subscripts refer to two members of an isotopic series. Multiplying Eq. (3) by $\mathbf{P}_{x}$ gives for both isotopically related molecules

$$
\begin{aligned}
& \mathbf{P}_{X} \mathbf{A}_{1}=\mathbf{P}_{X} \mathbf{A}_{\mathrm{g}}-\mathbf{P}_{X} \boldsymbol{\alpha}_{1} \boldsymbol{\beta}_{1} \mathbf{A}_{\mathrm{g}}, \\
& \mathbf{P}_{X} \mathbf{A}_{2}=\mathbf{P}_{X} \mathbf{A}_{\mathrm{g}}-\mathbf{P}_{X} \boldsymbol{\alpha}_{2} \boldsymbol{\beta}_{2} \mathbf{A}_{\mathrm{g}} .
\end{aligned}
$$

Equations (15) and (16) reveal

$$
-\mathbf{P}_{X} \boldsymbol{\alpha}_{1} \boldsymbol{\beta}_{1} \mathbf{A}_{2}=-\mathbf{P}_{X} \boldsymbol{\alpha}_{1} \boldsymbol{\beta}_{1} \mathbf{A}_{\mathrm{g}}+\mathbf{P}_{X} \boldsymbol{\alpha}_{2} \boldsymbol{\beta}_{2} \mathbf{A}_{\mathrm{g}} .
$$

The left-hand side of Eq. (17) is the relative rotational correction, denoted by $\mathbf{V}_{12}(3)$, while the right-hand side represents the difference of two absolute corrections, thus showing that the relative correction equals the difference of the two corresponding absolute ones:

$$
\mathrm{V}_{\mathrm{i2}}=\mathrm{V}_{1}-\mathrm{v}_{2} \text {. }
$$

It should be noted, however, that Eqs. (15)-(18) are valid only if parallel Cartesian axis frames are used for all modes that will be compared. Since the principal-axis system may be translated and rotated upon isotopic substitution, all principal-axis systems should be rotated to an orientation parallel to a chosen reference system (e.g., the principal-axis system of the parent molecule (5)). Although not explicitly mentioned by Crawford (4), this requirement obviously holds for absolute as well as relative corrections. Only if this requirement is satisfied is the $\mathbf{P}_{X}$ polar tensor the same for all molecules of the isotopic series, allowing corresponding $\mathbf{P}_{\mathrm{R}}$-tensor elements to be compared and corrected.

If the intensities belonging to the modes of equal groups in different (not isotopically related) molecules have to be compared, the corresponding groups should be given an equivalent position within the Cartesian frame.

\section{DISCUSSION}

\section{Rotational Corrections for Stretching Modes}

As is clear from the analytical expressions for the appropriate elements of the $\boldsymbol{\beta} \mathbf{A}_{\mathrm{Rg}}$ matrix the amount of rotation caused by a pure geometrical stretching mode is independent of the displacements actually chosen. This clearly demonstrates the uniqueness of the rotational contributions involved in the observed dipole moment derivatives. Since the $\boldsymbol{\rho}$-vectors describing a pure geometrical stretching mode never result in a rotation of any bond of the molecule but merely cause parallel shifts of the bonds not involved in the stretching mode, it becomes clear that rotational corrections for stretching modes can be obtained from the bond direction changes emerging from the $A$ matrix $(6,7)$.

The use of heavy isotopes for the calculation of absolute corrections for stretch- 
ing modes is based on the fact that the $\mathbf{A}$ matrix columns corresponding to the bonds adjacent to the heavy isotopes turn out to be (almost) equal to the corresponding columns of the $\boldsymbol{A}_{\mathrm{g}}$ matrix (with $k_{11}=1$ and $k_{13}=0$, see Eqs. (7)-(11)). If molecule 2 contains the heavy isotopes Eq. (17) reads

$$
\mathbf{V}_{12}=-\mathbf{P}_{X} \boldsymbol{\alpha}_{1} \boldsymbol{\beta}_{1} \mathbf{A}_{2}=-\mathbf{P}_{X} \boldsymbol{\alpha}_{1} \boldsymbol{\beta}_{1} \mathbf{A}_{\mathrm{g}}=\mathbf{V}_{\mathrm{t}}
$$

Thus with a heavy isotope reference molecule the relative rotational corrections are equal to the absolute ones for the stretching modes corresponding to bonds adjacent to the heavy isotopes.

\section{Rotational Corrections for Bending Modes}

It is important to note that the dipole moment change emerging from a pure geometrical angle bending mode depends not only on the bond moments of the bonds having the apex atom of the angle in common but also on the electrical structure of those parts of the molecule that are attached to both primary bonds. Only the remaining part of the molecule that is attached to the apex atom does not contribute to the molecular dipole moment change (in zero-order approximation) since the atoms of that part of the molecule including the apex atom are not displaced in a pure geometrical bending mode. The concept of rotational corrections is based on the idea that the intensities of equivalent modes in different molecules can be made comparable by correcting them for the contributions resulting from the Eckart conditions. This raises the question Which modes are equivalent? In zero-order approximation the answer is simple for stretching modes: Identical bonds lead to equivalent modes. The subsequent question How identical are identical bonds? refers to first- and higher-order effects, and answering that question is the very aim of comparisons of the corrected intensities of zero-order identical modes. The influence of first- and higher-order effects on the intensities determines the extent of transferability of intensity parameters.

For bending modes to be equivalent within the zero-order approximation requires not only that the primary bonds be identical in both molecules but also those parts of both molecules that are attached to the primary bonds (obviously apart from isotope substitution). Again, the question How identical are equivalent bending modes in view of the different atoms attached to the apex atom? has to be investigated by comparing the intensities corrected for the rotational contributions.

\section{APPLICATIONS}

\section{HOF and DOF}

The absolute rotational corrections for HOF and DOF have been calculated according to Eq. (6). For each molecule the calculations were performed within the principal-axis system, leading to the values of Table $I$. The $V$ tensor of HOF has been rotated in order to find the corrections for HOF in the DOF-axis system. These values are also given in lable 1. Furthermore, relative rotational corrections have been calculated directly from the Crawford expression as well as from the difference between the absolute values. These values have been calculated within the DOF-principal-axis system. Both calculations lead to exactly the same values 
for the relative corrections. The calculated values are given in Table I. Taking HOF as molecule 1 and DOF as molecule 2, the expressions for the absolute and relative corrections read:

$$
\begin{aligned}
\mathbf{V}_{1} & =-\mathbf{P}_{\rho 1} \boldsymbol{\beta}_{1} \mathbf{A}_{\mathrm{g} 1}, \\
\mathbf{V}_{2} & =-\mathbf{P}_{\rho 2} \boldsymbol{\beta}_{2} \mathbf{A}_{\mathrm{g} 2}, \\
\mathbf{V}_{21} & =\mathbf{V}_{2}-\mathbf{T} \mathbf{V}_{1}, \\
\mathbf{V}_{21} & =-\mathbf{P}_{\rho 2} \boldsymbol{\beta}_{2} \mathbf{T} \mathbf{A}_{1},
\end{aligned}
$$

where subscript 1 refers both to the HOF molecule and to the HOF-principal-axis system. The same holds for subscript 2 . The transformation matrix $\mathbf{T}$ rotates the HOF-principal-axis system to a position parallel to the corresponding axes of the DOF frame. Both for HOF and DOF the following geometrical and electrical parameters have been used: $r_{\mathrm{OH}}=0.964 \AA, r_{\mathrm{OF}}=1.442 \AA, \phi(\mathrm{HOF})=97.2^{\circ}(8)$, $\mu_{x}=-0.37 \mathrm{D}$, and $\mu_{z}=-2.20 \mathrm{D}(9-11)$. Since the dipole moment components given by Rock et al. (9) for HOF and DOF are not internally consistent due to experimental uncertainties, we have used the electrical structure of HOF for both molecules. The dipole moment vector points from the center of negative charge to the center of positive charge. The signs of the dipole moment components have been chosen in accordance with the results reported in Refs. $(10,11)$. The positions of both molecules in the principal-axis system are given in Fig. 1.

\section{TABLE I}

Absolute and Relative Rotational Corrections for HOF and DOF

\begin{tabular}{ccccccc}
\hline$v_{1}$ (HOF) & HOF-frame & & \multicolumn{3}{c}{$\mathrm{TV}$ (HOF) } & DOF-frame \\
& $\mathrm{R}_{1}$ & $\mathrm{R}_{2}$ & $\mathrm{R}_{3}$ & $\mathrm{R}_{1}$ & $\mathrm{R}_{2}$ & $\mathrm{R}_{3}$ \\
$\mathrm{x}$ & -0.0847 & 0.0567 & -0.9883 & -0.0840 & 0.0562 & -0.9800 \\
$\mathrm{y}$ & 0 & 0 & 0 & 0 & 0 & 0 \\
$z$ & 0.0143 & -0.0095 & 0.1662 & 0.0180 & -0.0120 & 0.2096
\end{tabular}
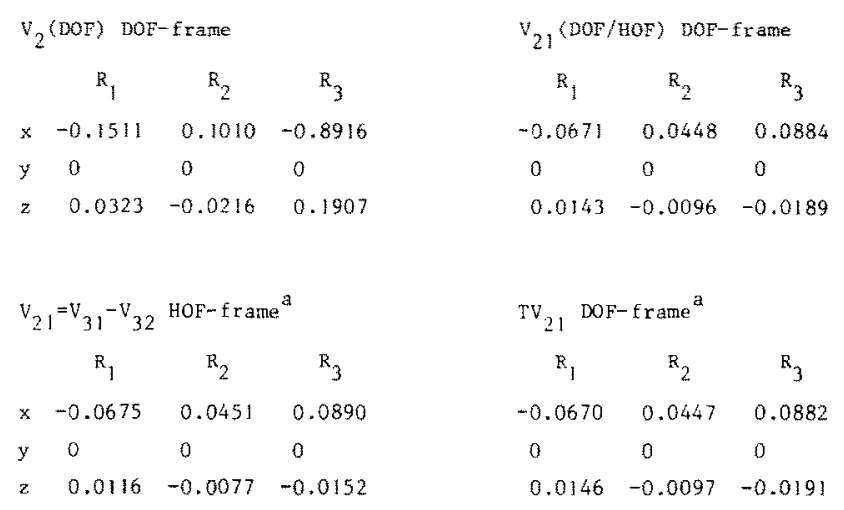

Note. Units: $\mathrm{D} \AA^{-1}$.

"Calculated from Ref. 12. Subscript 3 refers to the unit mass molecule. See text. 


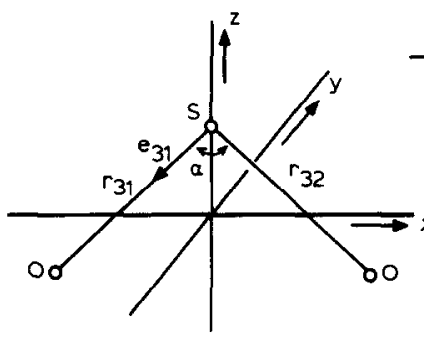

a

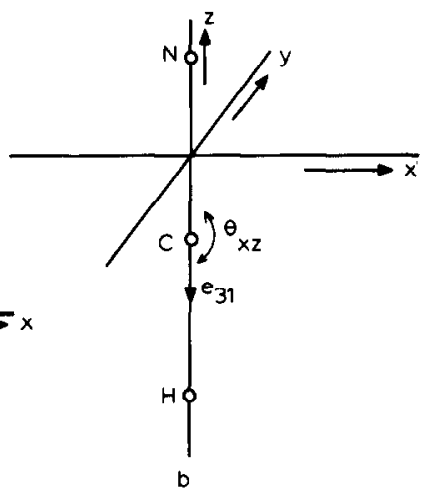

FIG. 2. Cartesian axes, internal coordinates, and bond unit vectors for (a) $\mathrm{SO}_{2}$ and (b) $\mathrm{HCN}$.

Using the same parameters Gussoni and Abbate (12) calculated rotational corrections for HOF and DOF relative to a unit mass molecule. Since Eq. (18) implies that within the same Cartesian frame

$$
\mathbf{V}_{21}=\mathbf{V}_{31}-\mathbf{V}_{32} \text {, }
$$

the corrections for HOF relative to DOF can be obtained from the corrections for HOF and DOF relative to the unit mass molecule. Since the relative corrections obtained from the paper of Gussoni and Abbate refer to the HOF frame they have to be transformed to the DOF frame in order to be comparable to our values. After

TABLE II

Absolute and Relative Rotational Corrections for $\mathrm{S}^{16} \mathrm{O}^{16} \mathrm{O}, \mathrm{S}^{18} \mathrm{O}^{18} \mathrm{O}$, and $\mathrm{S}^{16} \mathrm{O}^{18} \mathrm{O}$

\begin{tabular}{|c|c|c|c|c|c|c|}
\hline \multicolumn{4}{|c|}{$v_{1}\left(\mathrm{SO}_{2} 16,16\right) \quad 16,16$-frame } & \multicolumn{3}{|c|}{$\mathrm{v}_{2}\left(\mathrm{SO}_{2}, 18,18\right) \quad 16,16$-frame } \\
\hline & $\mathrm{R}_{1}$ & $\mathrm{R}_{2}$ & $R_{3}$ & $\mathrm{R}_{1}$ & $\mathbf{R}_{2}$ & $\mathrm{R}_{3}$ \\
\hline$x$ & 0.1406 & -0.1406 & 0 & 0.1502 & -0.1502 & 0 \\
\hline $\mathrm{y}$ & 0 & 0 & 0 & 0 & 0 & 0 \\
\hline $\mathrm{z}$ & 0 & 0 & 0 & 0 & 0 & 0 \\
\hline \multicolumn{2}{|c|}{$\mathrm{v}_{3}\left(\mathrm{So}_{2}, 16,18\right)$} & \multicolumn{2}{|c|}{16,18 -frame } & \multicolumn{3}{|c|}{$\mathrm{TV}_{3}\left(\mathrm{SO}_{2} 16,18\right) 16,16-\mathrm{frame}$} \\
\hline & $\mathrm{R}_{1}$ & $\mathrm{R}_{2}$ & $\mathrm{R}_{3}$ & $\mathrm{R}_{1}$ & $\mathrm{R}_{2}$ & $\mathrm{R}_{3}$ \\
\hline$x$ & 0.14523 & -0.14523 & 0.02652 & 0.14526 & -0.14526 & 0.02653 \\
\hline$y$ & 0 & 0 & 0 & 0 & 0 & 0 \\
\hline$z$ & 0.00291 & -0.00291 & 0.00053 & 0 & 0 & 0 \\
\hline \multicolumn{2}{|c|}{$v_{12}$} & \multicolumn{2}{|c|}{$16,16-$ frame } & $v_{13}$ & \multicolumn{2}{|c|}{16,16 -frame } \\
\hline & $\mathbf{R}_{1}$ & $\mathrm{R}_{2}$ & $\mathrm{R}_{3}$ & $\mathrm{R}_{1}$ & $\mathrm{R}_{2}$ & $\mathrm{R}_{3}$ \\
\hline $\mathbf{x}$ & -0.0096 & 0.0096 & 0 & -0.0046 & 0.0046 & -0.0265 \\
\hline y & 0 & 0 & 0 & 0 & 0 & 0 \\
\hline $\mathrm{z}$ & 0 & 0 & 0 & 0 & 0 & 0 \\
\hline
\end{tabular}

Note. Units: $\mathrm{D} \AA$. 
TABLE III

Absolute and Relative Rotational Corrections for the Bending Mode of HCN and DCN ${ }^{\text {a }}$

\begin{tabular}{llll}
\hline & $V_{1}\left(R_{3}\right)$ & $V_{2}\left(R_{3}\right)$ & $V_{12}\left(R_{3}\right)$ \\
\hline This work & -1.0217 & -0.7623 & -0.2594 \\
From Ref. (12) & & & -0.1881 \\
\hline
\end{tabular}

Note. Units: D $\AA^{-1}$.

transformation to the DOF frame and taking into account the differences in axes directions used by Gussoni and Abbate and by us, the relative corrections calculated from their data correspond very well to our values (see Table I).

$S^{16} O^{16} \mathrm{O}, S^{18} \mathrm{O}^{18} \mathrm{O}$ and $\mathrm{S}^{16} \mathrm{O}^{18} \mathrm{O}$

The absolute and relative corrections for $\mathrm{SO}_{2}(16,16), \mathrm{SO}_{2}(18,18)$, and $\mathrm{SO}_{2}(16,18)$ have been calculated. The internal coordinates (2) and Cartesian axes are indicated in Fig. 2a. The following geometrical parameters have been used: $r_{\mathrm{S} o}$ $=1.4308 \AA, \phi\left(\mathrm{SO}_{2}\right)=119.317^{\circ}(13)$. The $z$-component of the permanent moment has been taken as $+1.61 \mathrm{D}(6)$. The calculated $\mathrm{V}$ tensors are collected in Table II. Subscripts 1, 2, and 3 refer to the $(16,16),(18,18)$, and $(16,18)$ molecules, respectively. The relative corrections obtained from the absolute corrections lead to exactly the same values as those obtained from the Crawford relation given by $\mathrm{Eq}$. (15). The elements of $\mathbf{V}_{1}$ lead on symmetry coordinate basis to a correction for the asymmetrical stretching mode of $+0.1989 \mathrm{D} \AA^{-1}$, in full accordance with the values obtained from the heavy isotope method and the bond direction changes $(3,6)$.

\section{$H C N$ and $D C N$}

The absolute and relative rotational corrections for the bending mode of HCN and DCN are given in Table III. The internal coordinates and Cartesian axes are indicated in Fig. 2b. The geometrical parameters are: $r_{\mathrm{CH}}=1.0659 \AA, r_{\mathrm{CN}}$ $=1.1531 \AA(14)$. The $z$-component of the permanent moment is $-2.95 \mathrm{D}(14)$. The internal coordinates are taken as

$$
\begin{array}{ll}
R_{1}=\Delta \mathrm{r}_{C H}, & R_{3}=\Delta \Theta_{x z}, \\
R_{2}=\Delta \mathrm{r}_{C H}, & R_{4}=\Delta \Theta_{y z} .
\end{array}
$$

The $V$ tensor subscripts 1 and 2 refer to $\mathrm{HCN}$ and DCN, respectively. Obviously $\mathrm{V}\left(R_{3}\right)=\mathrm{V}\left(R_{4}\right)$, while $\mathrm{V}\left(R_{1}\right)=\mathrm{V}\left(R_{2}\right)=\mathbf{0}$. The relative correction $\mathrm{V}_{12}\left(R_{3}\right)$ has been calculated from $V_{1}-V_{2}$ as well as from Eq. (15). Both calculations lead to the same value. Gussoni and Abbate (12) reported the rotational correction for the $R_{3}$ mode relative to a unit mass molecule. From their values we have also calculated $V_{12}$ according to Eq. (24). The resulting value is given in Table III. The considerable difference from our value suggests an error in the values of Ref. (12). 


\section{ACKNOWLEDGMENT}

We gratefully acknowledge fruitful discussions with Dr. F. G. Dijkman.

RECEIVED: October 5, 1978

\section{REFERENCES}

1. S. R. Polo, J. Chem. Phys. 24, 1133-1138 (1956).

2. J. H. G. Bode, W. M. A. SMit, AND A. J. van Straten, J. Mol. Spectrosc. 75, 478-484 (1979).

3. A. J. van Straten and W. M. A. Smit, $J$, Mol. Spectrose. 56, 484-493 (1975).

4. B. L. Crawford, JR., J. Chem. Phys. 20, 977-981 (1952).

5. W. M. A. Smit, J. H. G. Bode, And A. J. van Straten, J. Mol. Spectrose. 72, 180-181 (1978).

6. A. J. van Straten and W. M. A. SMrt, J. Mol. Spectrose. 62, 297-312 (1976).

7. A. J. van Straten and W. M. A. SMit, J. Mol. Spectrosc. 65, 202-218 (1977).

8. H. Kim, E. F. Pearson, and E. H. Appelman, J. Chem. Phys. 56, 1-3 (1972); E. F. Pearson AND H. Kim, $J$. Chem. Phys. 57, 4230-4233 (1972).

9. S. L. Rock, E. F. Pearson, E. H. Appelman, C. L. Norris, and W. H. Flygare, J. Chem. Phys. 59, 3940-3945 (1973).

10. J. C. Hindman, A. Svirmickas, and E. H. Appelman, J. Chem. Phys. 57, 4542-4543 (1972).

11. H. Kim AND J. R. SABIN, Chem. Phys. Lett. 20, 215-218 (1973).

12. M. Gussoni and S. AbBate, J. Mol. Spectrose. 62, 53-59 (1976).

13. Y. Morino, Y. Kikuchi, S. SaIto, And E. Hirota, I. Mol. Spectrose. 13, 95-118 (1964).

14. R. E. Bruns and W. B. Person, J. Chem. Phys. 53, 1413-1417 (1970). 